\title{
AMENABILITY CONSTANTS FOR SEMILATTICE ALGEBRAS
}

\author{
MAHYA GHANDEHARI, HAMED HATAMI AND NICO SPRONK
}

\begin{abstract}
For any finite commutative idempotent semigroup $S$, a semilattice, we show how to compute the amenability constant of its semigroup algebra $\ell^{1}(S)$, which is always of the form $4 n+1$. We then show that these give lower bounds to amenability constants of certain Banach algebras graded over semilattices. We also demonstrate an example of a commutative Clifford semigroup $G$ for which amenability constant of $\ell^{1}(G)$ is not of the form $4 n+1$. We also show there is no commutative semigroup with amenability constant between 5 and 9 .
\end{abstract}

\section{I}

In conjunction with $\mathrm{V}$. Runde [13, the third named author proved that for a locally compact group $G, G$ is compact if and only if its Fourier-Stieltjes algebra $\mathrm{B}(G)$ is operator amenable with operator amenability constant less than 5. In a subsequent article [14, examples of non-compact groups $G_{1}$ were found for which the operator amenability constant is exactly 5 . In related work of Dales, Lau and Strauss [3, Corollary 10.26], improving on [16, Theorem 3.2], it was shown that a semigroup algebra $\ell^{1}(S)$ has amenability constant less than 5 , if and only if $S$ is an amenable group. For the multiplicative semigroup $L_{1}=\{0,1\}$, it is known that the amenability constant of $\ell^{1}\left(L_{1}\right)$ is 5 . These parallel facts are not coincidences since for the special groups $G_{1}$, mentioned above, $\mathrm{B}\left(G_{1}\right)$ is $\ell^{1}$-graded over $L_{1}$, i.e. there are 1-operator amenable subalgebras $\mathcal{A}_{0}$ and $\mathcal{A}_{1}$ such that $\mathrm{B}\left(G_{1}\right)=\mathcal{A}_{0} \oplus_{\ell^{1}} \mathcal{A}_{1}$, and $\mathcal{A}_{0}$ is an ideal.

We are thus led to consider the general situation of Banach algebras graded over semilattices, i.e. commutative idempotent semigroups, which we define in Section 2, To do this, in Section 1 we develop a method for computing the amenability constants associated to finite semilattice algebras. The results in Section 1 have a similar flavour to some results from those in the recent monograph [3, and are very similar to some results of Duncan and Namioka 4. However, our method is explicit and quantitative, and thus is a nice complement to their work. In Section 2 we obtain a lower bound for the amenability constant of Banach algebras graded over finite

2000 Mathematics Subject Classification. Primary 46H20, 43A20; Secondary 20M14, 43A30. Key words and phrases. amenable/contractible Banach algebra, semilattice, graded Banach algebra.

Research of the third named author supported by NSERC Grant 312515-05. 
semilattices. We show a surprising example which indicates our lower bound is not, in general the amenability constant. We show, at least for certain finite dimensional algebras graded over linear semilattices, that our lower bound is achieved. We close with an answer to a question asked of us by H.G. Dales: we show that there does not exist a commutative semigroup $G$ such that $5<\operatorname{AM}\left(\ell^{1}(G)\right)<9$.

There are natural examples of Banach algebras from harmonic analysis, due to Taylor [17, Inoue [9], and Ilie and Spronk [7, 8, to which our techniques apply. We recommend the reader to [7] and [13] for more on this. We feel that ideas developed here may lead to a tool to help classify which locally compact groups admit operator amenable Fourier-Stieltjes algebras $\mathrm{B}(G)$. Our hope is that the operator amenability constants $\operatorname{AM}_{o p}(\mathrm{~B}(G))$ can all be computed. We conjecture they are a subset of $\{4 n+1: n \in \mathbb{N}\}$, motived by Theorem 1.7 and Theorem 2.2 , below. We hope that these values will serve as a tool for classifying for which groups $G, \mathrm{~B}(G)$ is operator amenable.

Interest in amenability of semigroup algebras, in particular for inverse semigroups and Clifford semigroups, goes back at least as far as Duncan and Namioka [4]. Grønbæk [5] characterised commutative semigroups $G$ for which $\ell^{1}(G)$ is amenable. A recent extensive treatise on $\ell^{1}$-algebras of semigroups has been written by Dales, Lau and Strauss [3, which includes a charaterisation of all semigroups $G$ for which $\ell^{1}(G)$ is amenable. Biflatness of $\ell^{1}(S)$, for a semilattice $S$, has recently been characterised by Choi [1].

0.1. Preliminaries. Let $\mathcal{A}$ be a Banach algebra. Let $\mathcal{A} \otimes^{\gamma} \mathcal{A}$ denote the projective tensor product. We let $m: \mathcal{A} \otimes^{\gamma} \mathcal{A} \rightarrow \mathcal{A}$ denote the multiplication map and we have left and right module actions of $\mathcal{A}$ on $\mathcal{A} \otimes^{\gamma} \mathcal{A}$ given on elementary tensors by

$$
a \cdot(b \otimes c)=(a b) \otimes c \text { and }(b \otimes c) \cdot a=b \otimes(c a) .
$$

A bounded approximate diagonal (b.a.d.) is a bounded net $\left(D_{\alpha}\right)$ in $\mathcal{A} \otimes^{\gamma} \mathcal{A}$ such that $\left(m\left(D_{\alpha}\right)\right)$ is a bounded approximate identity in $\mathcal{A}$, i.e.

$$
\lim _{\alpha} a m\left(D_{\alpha}\right)=a \text { and } \lim _{\alpha} m\left(D_{\alpha}\right) a=a \text { for each } a \text { in } \mathcal{A}
$$

and $\left(D_{\alpha}\right)$ is asymptotically central for the $\mathcal{A}$-actions, i.e.

$$
\lim _{\alpha}\left(a \cdot D_{\alpha}-D_{\alpha} \cdot a\right)=0 \text { for each } a \text { in } \mathcal{A} \text {. }
$$

Following Johnson [10], we will say that a Banach algebra $\mathcal{A}$ is amenable if it admits a b.a.d. A quantitative feature of amenability was introduced by Johnson in [11], for applications to Fourier algebras of finite groups. The amenability constant of an amenable Banach algebra $\mathcal{A}$ is given by

$$
\operatorname{AM}(\mathcal{A})=\inf \left\{\sup _{\alpha}\left\|D_{\alpha}\right\|_{\gamma}:\left(D_{\alpha}\right) \text { is a b.a.d. for } \mathcal{A}\right\} .
$$

The problem of understanding amenable semigroup algebras in terms of their amenability constants has attracted some attention [16, 3]. 
We call $\mathcal{A}$ contractible if it admits a diagonal, i.e. an element $D$ in $\mathcal{A} \otimes^{\gamma} \mathcal{A}$ for which

$$
\begin{gathered}
a m(D)=a=m(D) a \text { and } \\
a \cdot D=D \cdot a
\end{gathered}
$$

for each $a$ in $\mathcal{A}$. Note, in particular, then $\mathcal{A}$ must be unital and the norm of the unit is bounded above by $\operatorname{AM}(\mathcal{A})$.

If $\mathcal{A}$ is a finite dimensional amenable Banach algebra, then $\mathcal{A} \otimes^{\gamma} \mathcal{A}$ is a finite dimensional Banach space, so any b.a.d. admits a cluster point $D$. Since any subnet of a b.a.d. is also a b.a.d., the cluster point must be a diagonal, whence $\mathcal{A}$ is contractible.

We record the following simple observation.

Proposition 0.1. If $\mathcal{A}$ is a contractible commutative Banach algebra, then the diagonal is unique.

Proof. We note that $\mathcal{A} \otimes^{\gamma} \mathcal{A}$ is a Banach algebra in an obvious way: ( $a \otimes$ $b)(c \otimes d)=(a c) \otimes(b d)$. If $D$ is a diagonal, then $(a \otimes b) D=a \cdot D \cdot b=(a b) \cdot D$ for $a, b$ in $\mathcal{A}$, by commutativity. Hence if $D^{\prime}$ is another diagonal

$$
D^{\prime} D=m\left(D^{\prime}\right) \cdot D=1 \cdot D=D
$$

and, similarly, $D^{\prime} D=D D^{\prime}=D^{\prime}$.

It will also be useful to observe the following.

Proposition 0.2. Let $\mathcal{A}$ and $\mathcal{B}$ be contractible Banach algebras, with respective diagonals $D_{\mathcal{A}}$ and $D_{\mathcal{B}}$, then $\mathcal{A} \otimes^{\gamma} \mathcal{B}$ has diagonal

$$
D_{\mathcal{A}} \otimes D_{\mathcal{B}} \in\left(\mathcal{A} \otimes^{\gamma} \mathcal{A}\right) \otimes^{\gamma}\left(\mathcal{B} \otimes^{\gamma} \mathcal{B}\right) \cong\left(\mathcal{A} \otimes^{\gamma} \mathcal{B}\right) \otimes^{\gamma}\left(\mathcal{A} \otimes^{\gamma} \mathcal{B}\right) .
$$

Proof. It is simple to check the diagonal axioms (0.3) and (0.4).

\section{Amenability constants for semilattice algebras}

A semilattice is a commutative semigroup $S$ in which each element is idempotent, i.e. if $s \in S$ then $s s=s$. If $s, t \in S$ we write

$$
s \leq t \quad \Leftrightarrow \quad s t=s
$$

It is clear that this defines a partial order on $S$. We note that if $S$ is a finite semilattice, then $o=\prod_{s \in S} s$ is a minimal element for $S$ with respect to this partial order. We note that if $S$ has a minimal element, then it is unique. Also if $S$ has a unit 1, then 1 is the maximal element in $S$.

A basic example of a semilattice is $\mathcal{P}(T)$, the set of all subsets of a set $T$, where we define $\sigma \tau=\sigma \cap \tau$ for $\sigma, \tau$ in $\mathcal{P}(T)$. The minimal element is $\varnothing$, and the maximal element is $T$. We call any subsemilattice of a semilattice $\mathcal{P}(T)$ a subset semilattice. This type of semilattice is universal as we have a semilattice "Cayley Theorem": for any semilattice $S$, the map $s \mapsto\{t \in$ $S: t \leq s\}: S \rightarrow \mathcal{P}(S)$ (or $s \mapsto\{t \in S \backslash\{o\}: t \leq s\}: S \rightarrow \mathcal{P}(S \backslash\{o\})$ ) is an injective semilattice homomorphism (by which $o \mapsto \varnothing$ ). 
For any semilattice $S$ we define

$$
\ell^{1}(S)=\left\{x=\sum_{s \in S} x(s) \delta_{s}: \text { each } x(s) \in \mathbb{C} \text { and }\|x\|_{1}=\sum_{s \in S}|x(s)|<\infty\right\}
$$

where each $\delta_{s}$ is the usual "point mass" function. Then $\ell^{1}(S)$ is a commutative Banach algebra under the norm $\|\cdot\|_{1}$ with the product

$$
\left(\sum_{s \in S} x(s) \delta_{s}\right) *\left(\sum_{t \in S} x(t) \delta_{t}\right)=\sum_{r \in S}\left(\sum_{s t=r} x(s) y(t)\right) \delta_{r} .
$$

In particular we have $\delta_{s} * \delta_{t}=\delta_{s t}$. We shall consider the Banach space $\ell^{\infty}(S)$, of bounded functions from $S$ to $\mathbb{C}$ with supremum norm, to be an algebra under usual pointwise operations. The Cayley map, indicated above, extends to an algebra homomorphism $\Sigma: \ell^{1}(S) \rightarrow \ell^{\infty}(S)$, given on each $\delta_{s}$ by

$$
\Sigma\left(\delta_{s}\right)=\chi_{\{t \in S: t \leq s\}}
$$

and extended linearly and continuously to all of $\ell^{1}(S)$. Here, $\chi_{T}$ is the indicator function of $T \subset S$. The map $\Sigma$ is called the Schützenburger map; see [1, §4] and references therein.

We note that if $S$ is finite, then $\Sigma$ is a bijection. In this case a formula for its inverse is given by

$$
\Sigma^{-1}\left(\chi_{s}\right)=\sum_{t \leq s} \mu(t, s) \delta_{t}
$$

where $\chi_{s}=\chi_{\{s\}}$ and $\mu:\{(t, s): S \times S: t \leq s\} \rightarrow \mathbb{R}$ is the Möbius function of the partially ordered set $(S, \leq)$ as defined in [15, §3.7]. Our computations in this section will be equivalent to explicitly computating $\mu$, though we will never need to know $\mu$ directly.

It follows from [4, Theorem 10] that $\ell^{1}(S)$ is amenable if and only if $S$ is finite. Thus it follows (0.3) that $\ell^{1}(S)$ is unital if $S$ is finite. If $S$ is unital, then $\delta_{1}$ is the unit for $\ell^{1}(S)$. If $S$ is not unital, the unit is more complicated. We let $M(S)$ denote the set of maximal elements in $S$ with respect to the partial ordering (1.1).

Proposition 1.1. If $S$ is a finite semilattice then the unit is given by $u=$ $\sum_{p \in S} u(p) \delta_{p}$ where

$$
u(p)=1-\sum_{t>p} u(t)
$$

for each $p$ in $S$ and we adopt the convention that an empty sum is 0 . Moreover

$$
\sum_{s \in S} u(s)=1
$$


Proof. While we have already established existence of the unit above, let us note that we can gain a very elementary proof of its existence. Indeed since $\Sigma: \ell^{1}(S) \rightarrow \ell^{\infty}(S)$ is a bijection, $u=\Sigma^{-1}\left(\chi_{S}\right)$ is the unit for $\ell_{1}(S)$.

If $p \in S$ then

$$
\delta_{p}=\delta_{p} * u=\left(\sum_{s \geq p} u(s)\right) \delta_{p}+\sum_{s<p}\left(\sum_{\substack{t \in S \\ t p=s}} u(t)\right) \delta_{s} .
$$

and thus, inspecting the coefficient of $\delta_{p}$, we obtain (1.4). Note that if $p \in M(S)$ the formula above gives $u(p)=1$, and for any $s$ in $S \backslash M(S)$ we have $\sum_{t \in S, t p=s} u(t)=0$. Thus, if we select $p$ in $M(S)$ we have

$$
\sum_{s \in S} u(s)=u(p)+\sum_{s<p}\left(\sum_{\substack{t \in S \\ t p=s}} u(t)\right)=1
$$

and thus obtain (1.5).

We note that if $S$ is a finite semilattice then $S \backslash M(S)$ is a subsemilattice, in fact an ideal, of $S$. We also note that $S \times S$ is also a semilattice and the partial order there satisfies

$$
(s, t) \leq(p, q) \quad \Leftrightarrow \quad s \leq p \text { and } t \leq q .
$$

The following gives an algorithm for computing the diagonal for $\ell^{1}(S)$.

Lemma 1.2. Let $S$ be a finite semilattice. Then the diagonal

$$
D=\sum_{(s, t) \in S \times S} d(s, t) \delta_{s} \otimes \delta_{t}
$$

satisfies, for all $(p, q)$ in $S \times S$,

(a) $d(p, p)=u(p)-\sum_{\substack{(s, t)>(p, p) \\ s t=p}} d(s, t)$;

(b) if $q \nsupseteq p$, then $d(p, q)=-\sum_{t>q} d(p, t)$ and $d(q, p)=-\sum_{s>q} d(s, p)$; and

(c) $d(p, q)=d(q, p)$.

Thus, each $d(p, q)$ is an integer, and for distinct elements $p, q$ in $M(S)$ we have $d(p, p)=1$ and $d(p, q)=0$.

Proof. The equation (0.3) gives us

$$
\sum_{p \in S} u(p) \delta_{p}=u=\sum_{(s, t) \in S \times S} d(s, t) \delta_{s t}=\sum_{p \in S}\left(\sum_{\substack{(s, t) \in S \times S \\ s t=p}} d(s, t)\right) \delta_{p}
$$


Since $s t=p$ necessitates $(s, t) \geq(p, p)$, we examine the coefficient of $\delta_{p}$ to find

$$
u(p)=\sum_{\substack{(s, t) \geq(p, p) \\ s t=p}} d(s, t)
$$

from which we obtain (a). In particular, if $p \in M(S)$ we obtain an empty sum in (a) and find $d(p, p)=1$. The equation (0.4) implies that $\delta_{q} \cdot D=D \cdot \delta_{q}$ and hence we obtain

$$
\sum_{(s, t) \in S \times S} d(s, t) \delta_{q s} \otimes \delta_{t}=\sum_{(s, t) \in S \times S} d(s, t) \delta_{s} \otimes \delta_{t q} .
$$

If $q \nsupseteq p$ then there is no $s$ in $S$ for which $q s=p$. Hence examining the coefficient of $\delta_{p} \otimes \delta_{q}$ and $\delta_{q} \otimes \delta_{p}$, respectively, in (1.8), yields

$$
0=\sum_{t \geq q} d(p, t) \quad \text { and } \quad \sum_{s \geq q} d(s, p)=0 .
$$

Hence we have established (b). In particular, if $q, p \in M(S)$ we have an empty sum in (b), so $d(p, q)=0$.

We can see for any pair $(p, q)$ with $p \neq q$, so $p \not \leq q$ or $q \not \leq p$, that $d(p, q)$ is determined by coefficients $(s, t)>(p, q)$. Hence by induction, using the coeficients $d(p, p)$ and $d(p, q)$ for distict maximal $p, q$ as a base, we obtain (c). For example, if $q \in M(S \backslash M(S))$, then (b) implies for every $p>q$ that

$$
d(p, q)=-\sum_{t>q} d(p, t)=-d(p, p)=-1
$$

and, similarly, $d(q, p)=-1$.

It is clear, form the above induction, that each $d(p, q)$ is an integer.

Let us see how Lemma 1.2 allows us to compute the diagonal $D$ of $\ell^{1}(S)$ for a finite semilattice $S$.

Step 1. We inductively define

$$
S_{0}=S, S_{1}=S \backslash M(S), \ldots, S_{k+1}=S_{k} \backslash M\left(S_{k}\right)
$$

and we let $n(S)=\min \left\{k: S_{k+1}=\varnothing\right\}$, so $S_{n(S)}=\{o\}$ and $S_{n(S)+1}=\varnothing$.

Step 2. We label $S=\left\{s_{0}, s_{1}, \ldots, s_{|S|-1}\right\}$ in any manner for which

$$
i \geq j \text { and } s_{i} \in S_{k} \quad \Rightarrow \quad s_{j} \in S_{k} .
$$

Thus, the elements of $M\left(S_{k}\right)$ comprise the last part of the list of $S_{k}$ for $k=1, \ldots, n(S)$. In particular, $s_{0}=o$ and $s_{|S|-1} \in M(S)$.

Step 3. The diagonal $D$ will be represented by an $|S| \times|S|$ matrix $[D]=$ $\left[d\left(s_{i}, s_{j}\right)\right]$. The lower rightmost corner will be the $|M(S)| \times|M(S)|$ identity matrix. We can then proceed, using formulas (b) and (a) from the lemma above, to compute the remaining entries of the lower rightmost $(|M(S)|+$ $1) \times(|M(S)|+1)$ corner of $[D]$, etc., until we are done. 
In order to describe certain semilattices $S$, we define the semilattice graph $\Gamma(S)=(S, e(S))$, where the vertex set is $S$ and the edge set is given by ordered pairs

$e(S)=\{(s, t) \in S \times S: s>t$ and there is no $r$ in $S$ for which $s>r>t\}$.

To picture such a graph for a finite semilattice $S$ it is helpful to describe levels. Let $S_{0}, S_{1}, \ldots, S_{n(S)}$ be the sequence of ideals of $S$ given in (1.10). For $s$ in $S$ we let the level of $s$ be given by

$$
\lambda(s)=n(S)-k \text { where } s \in M\left(S_{k}\right) .
$$

Note that for the power set semilattice $\mathcal{P}(T), \lambda(\sigma)=|\sigma|$, the cardinality of $\sigma$. However, this relation need not hold for a subsemilattice of $\mathcal{P}(T)$, as is evident from the Example 1.4, below. A 6-element, 4-level semilattice is illustrated in (2.7).

We apply this algorithm to obtain the following examples. We denote, for a finite semilattice $S$, the amenability constant

$$
\operatorname{AM}(S)=\operatorname{AM}\left(\ell^{1}(S)\right)=\|D\|_{1}=\sum_{(s, t) \in S \times S}|d(s, t)|
$$

where we recall the well-known isometric identification $\ell^{1}(S) \otimes^{\gamma} \ell^{1}(S) \cong$ $\ell^{1}(S \times S)$.

Example 1.3. Let $L_{n}=\{0,1,2, \ldots, n\}$ be a "linear" semilattice with operation $s t=s \wedge t=\min \{s, t\}$. Then we obtain diagonal with $(n+1) \times(n+1)$ matrix

Hence $\operatorname{AM}\left(L_{n}\right)=4 n+1$.

$$
[D]=\left[\begin{array}{rrrrr}
2 & -1 & \ldots & 0 & 0 \\
-1 & 2 & \ddots & 0 & 0 \\
\vdots & \ddots & \ddots & \ddots & \vdots \\
0 & 0 & \ddots & 2 & -1 \\
0 & 0 & \ldots & -1 & 1
\end{array}\right]
$$

Example 1.4. Let $F_{n}=\left\{o, s_{1}, \ldots, s_{n}\right\}$ be the $n+1$ element "flat" semilattice with multiplications $s_{i} s_{j}=o$ if $i \neq j$. Then we obtain unit

$$
u=\delta_{s_{1}}+\cdots+\delta_{s_{n}}+(1-n) \delta_{o}
$$

and diagonal with $(n+1) \times(n+1)$-matrix

$$
[D]=\left[\begin{array}{rrrrr}
n+1 & -1 & -1 & \ldots & -1 \\
-1 & 1 & 0 & \ldots & 0 \\
-1 & 0 & 1 & \ddots & \vdots \\
\vdots & \vdots & \ddots & \ddots & 0 \\
-1 & 0 & \ldots & 0 & 1
\end{array}\right]
$$

Hence $\operatorname{AM}\left(F_{n}\right)=4 n+1$. 
Example 1.5. Let $F_{n}^{1}=\left\{o, s_{1}, \ldots, s_{n}, 1\right\}$ be the unitasation of $F_{n}$, above. Then we obtain diagonal with $(n+2) \times(n+2)$ matrix

$$
[D]=\left[\begin{array}{crrrc}
n^{2}-n+2 & -n & \ldots & -n & n-1 \\
-n & 2 & \ldots & 1 & -1 \\
\vdots & \vdots & \ddots & \vdots & \vdots \\
-n & 1 & \ldots & 2 & -1 \\
n-1 & -1 & \ldots & -1 & 1
\end{array}\right] .
$$

Hence $\operatorname{AM}\left(F_{n}^{1}\right)=4 n^{2}+4 n+1$.

The next example is less direct than the previous ones, so we offer a proof.

Example 1.6. Let $P_{n}=\mathcal{P}(\{1, \ldots, n\})$ with multiplication st $=s \cap t$. Then the diagonal $D$ has $2^{n} \times 2^{n}$ matrix which is, up to permutative similarity, the Kronecker product

$$
\left[\begin{array}{rr}
2 & -1 \\
-1 & 1
\end{array}\right] \otimes \cdots \otimes\left[\begin{array}{rr}
2 & -1 \\
-1 & 1
\end{array}\right] \text { (n times). }
$$

Hence $\operatorname{AM}\left(P_{n}\right)=5^{n}$.

Proof. If $s \in P_{n}$ let $\chi_{s}:\{1, \ldots, n\} \rightarrow\{0,1\}=L_{1}$ be its indicator function. It is easily verified that the map $s \mapsto \chi_{s}: P_{n} \rightarrow L_{1}^{n}$ is a semilattice isomorphism. Thus there is an isometric identification $\ell^{1}\left(P_{n}\right) \cong$ $\ell^{1}\left(L_{1}\right) \otimes^{\gamma} \cdots \otimes^{\gamma} \ell^{1}\left(L_{1}\right)$. Then it follows from Proposition 0.2 above that $D=D_{1} \otimes \cdots \otimes D_{1}$ where $D_{1}$ is the diagonal for $\ell^{1}\left(L_{1}\right)$, which, by the algorithm has matrix

$$
\left[D_{1}\right]=\left[\begin{array}{rr}
2 & -1 \\
-1 & 1
\end{array}\right]
$$

The amenability constant $\operatorname{AM}\left(P_{n}\right)$ can be easily computed by induction.

We have the following summary result.

Theorem 1.7. If $S$ is a finite semilattice, then $\operatorname{AM}(S)=4 n+1$ for some integer $n \geq 0$. All such numbers are achieved.

Proof. We first establish that for $p$ in $S, d(p, p) \geq 0$. This does not seem obvious from Lemma 1.2. We use a calculation from [1, §3] which exploits the Möbius function. We have that $\Sigma: \ell^{1}(S) \rightarrow \ell^{\infty}(S)$ is invertible and $\tilde{D}=\sum_{r \in S} \chi_{r} \otimes \chi_{r}$ is the diagonal for $\ell^{\infty}(S)$. Thus, using (1.3), we have that

$$
\begin{aligned}
D=\Sigma^{-1} \otimes \Sigma^{-1}(\tilde{D}) & =\sum_{r \in S}\left(\sum_{s \in S} \tilde{\mu}(s, r) \delta_{s}\right) \otimes\left(\sum_{t \in S} \tilde{\mu}(t, r) \delta_{t}\right) \\
& =\sum_{(s, t) \in S \times S}\left(\sum_{r \in S} \tilde{\mu}(s, r) \tilde{\mu}(t, r)\right) \delta_{s} \otimes \delta_{t}
\end{aligned}
$$


is the diagonal for $\ell^{1}(S)$, where $\tilde{\mu}(s, t)=\mu(s, t)$ if $s \leq t$ and $\tilde{\mu}(s, t)=0$, otherwise. Inspecting the coeficient of $\delta_{p} \otimes \delta_{p}$ we obtain

$$
d(p, p)=\sum_{r \in S} \tilde{\mu}(p, r)^{2} \geq 1>0
$$

since $\tilde{\mu}(p, p)=\mu(p, p)=1$ by [15, §3.7]. We now observe, using (1.7) and then $(1.5)$, that

$$
\sum_{(s, t) \in S \times S} d(s, t)=\sum_{p \in S} \sum_{\substack{s, t) \in S \times S \\ s t=p}} d(s, t)=\sum_{p \in S} u(p)=1 .
$$

By symmetry, if $p \neq q$ then $|d(p, q)|+|d(q, p)| \equiv d(p, q)+d(q, p) \bmod 4$. Hence we have

$$
\operatorname{AM}(S) \equiv \sum_{(s, t) \in S \times S}|d(s, t)| \equiv \sum_{(s, t) \in S \times S} d(s, t) \equiv 1 \quad \bmod 4 .
$$

Finally, Examples 1.3 and 1.4provide us with semilattices admitting amenability constants $4 n+1$, for each integer $n \geq 0$.

We now gain a crude lower bound for $\operatorname{AM}(S)$ which we will require for Proposition 2.4.

Corollary 1.8. For any finite semilattice $S$ we have $\operatorname{AM}(S) \geq 2|S|-1$.

Proof. We have from (1.11) that $d(p, p) \geq 1$ for each $p$ in $S$. It then follows from (1.9) that for $p>o$ we have $\sum_{t \geq o} d(p, t)=0$ from which we obtain $\sum_{t \neq p}|d(p, t)| \geq 1$. It then follows that

$$
\begin{aligned}
\operatorname{AM}(S)=\sum_{(s, t) \in S \times S}|d(s, t)| & \geq d(o, o)+\sum_{p>0}\left(d(p, p)+\sum_{t \neq p}|d(p, t)|\right) \\
& \geq 1+(|S|-1) 2
\end{aligned}
$$

and we are done.

We note that if $S$ is unital, then for $p<1, u(p)=0$ and since $d(s, t)=$ $d(t, s)$ for $(s, t)>(p, p)$ we find from Lemma 1.2 (a) that $d(p, p)$ is even; in particular $d(p, p) \geq 2$. The proof above may be adapted to show $\operatorname{AM}(S) \geq$ $4|S|-3$, in this case. We conjecture the estimate $\operatorname{AM}(S) \geq 4|S|-3$ holds for any finite semilattice $S$.

\section{BANACH ALGEBRAS GRADED OVER SEMILATTICES}

A Banach algebra $\mathcal{A}$ is graded over a semigroup $S$ if we have closed subspaces $\mathcal{A}_{s}$ for each $s$ in $S$ such that

$$
\mathcal{A}=\ell^{1}-\bigoplus_{s \in S} \mathcal{A}_{s} \text { and } \mathcal{A}_{s} \mathcal{A}_{t} \subset \mathcal{A}_{s t} \text { for } s, t \text { in } S .
$$


We will be interested strictly in the case where $S$ is a finite semilattice. Notice in this case each $\mathcal{A}_{s}$ is a closed subalgebra of $\mathcal{A}$. The next proposition can be proved by a straightforward adaptation of the proof of [14, Proposition 3.1]. However, we offer another proof.

Proposition 2.1. Let $S$ be a finite semilattice and $\mathcal{A}$ be graded over $S$. Then $\mathcal{A}$ is amenable if and only if each $\mathcal{A}_{s}$ is amenable.

Proof. Suppose $\mathcal{A}$ is amenable. If $s \in S$, then $\mathcal{A}^{s}=\bigoplus_{t \leq s} \mathcal{A}_{t}$ is an ideal in $\mathcal{A}$ which is complemented and hence an amenable Banach algebra (see [12, Theorem 2.3.7], for example). It is easy the check that the projection $\pi_{s}: \mathcal{A}^{s} \rightarrow \mathcal{A}_{s}$ is a quotient homomorphism. Hence it follows that if $\left(D_{\alpha}^{s}\right)$ is an approximate diagonal for $\mathcal{A}^{s}$ then $\left(\pi_{s} \otimes \pi_{s}\left(D_{\alpha}^{s}\right)\right)$ is an approximate diagonal for $\mathcal{A}_{s}$. (This is quotient argument is noted in [12, Corollary 2.3.2] and [3, Proposition 2.5].)

Now suppose that each $\mathcal{A}_{s}$ is amenable. Let $S_{0}, S_{1}, \ldots, S_{n(S)}$ be the sequence of ideals from (1.10). For each $n=0,1, \ldots, n(S)$ we set $\mathcal{A}_{n}=$ $\bigoplus_{s \in S_{n}} \mathcal{A}_{s}$ and observe, for each $n=0,1, \ldots, n(S)-1$, that we have an isometrically isomorphic identification

$$
\mathcal{A}_{n} / \mathcal{A}_{n+1}=\ell^{1}-\bigoplus_{s \in M\left(S_{n}\right)} \mathcal{A}_{s}
$$

where multiplication in the latter is pointwise, i.e. $\mathcal{A}_{s} \mathcal{A}_{t}=\{0\}$ if $s \neq$ $t$ in $M\left(S_{n}\right)$. The pointwise algebra $\ell^{1}-\bigoplus_{s \in M\left(S_{n}\right)} \mathcal{A}_{s}$ is amenable as each $\mathcal{A}_{s}$ is amenable; if $\left(D_{s, \alpha}\right)$ is a bounded approximate diagonal for each $\mathcal{A}_{s}$, then in

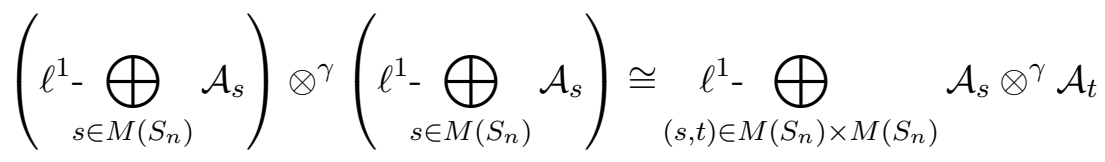

the net of elements $D_{\alpha}=\sum_{s \in M\left(S_{n}\right)} D_{s, \alpha}$ is an approximate diagonal. Thus if $\mathcal{A}_{n+1}$ is amenable, then $\mathcal{A}_{n}$ must be too by [12, Theorem 2.3.10]. The algebra $\mathcal{A}_{n(S)}=\mathcal{A}_{o}$ is amenable, and hence we may finish by an obvious induction.

In the computations which follow, we will require one of the following linking assumptions which are very natural for our examples.

(LA1) For each $s$ in $S$ there is a bounded approximate identity $\left(u_{s, \alpha}\right)_{\alpha}$ in $\mathcal{A}_{s}$, such that for each $t \leq s$ and $a_{t} \in \mathcal{A}_{t}$ we have $\lim _{\alpha} u_{s, \alpha} a_{t}=a_{t}=\lim _{\alpha} a_{t} u_{s, \alpha}$.

(LA2) For each $s \in S$ there is a contractive character $\chi_{s}: \mathcal{A}_{s} \rightarrow \mathbb{C}$ such that for each $s, t$ in $S, a_{s} \in \mathcal{A}_{s}$ and $a_{t} \in \mathcal{A}_{t}$, we have $\chi_{s t}\left(a_{s} b_{t}\right)=$ $\chi_{s}\left(a_{s}\right) \chi_{t}\left(a_{t}\right)$.

Notice that in (LA1), each $\left(u_{s, \alpha}\right)_{\alpha}$ is a bounded approximate identity for $\mathcal{A}^{s}=\ell^{1}-\bigoplus_{t \leq s} \mathcal{A}_{t}$. Thus since $\mathcal{A}^{s}$ is an $\mathcal{A}_{s}$-module, Cohen's factorisation 
theorem [6, 32.22] tells us that

(2.1) for each $a$ in $\mathcal{A}^{s}$ there is $v_{s} \in \mathcal{A}_{s}$ and $a^{\prime}$ in $\mathcal{A}^{s}$ such that $a=v_{s} a^{\prime}$.

There is a right factorisation analogue, and the result also holds on each $\mathcal{A}_{s}$ module $\mathcal{A}_{t}$, where $t \leq s$. We note that (LA2) is equivalent to having a contractive character $\chi: \mathcal{A} \rightarrow \mathbb{C}$ such that $\left.\chi\right|_{\mathcal{A}_{s}}=\chi_{s}$ for each $s$.

We note that many natural Banach algebras, graded over semilattices, which arise in harmonic analysis, satisfy (LA2). However, (LA1) can be used whenever each component algebra $\mathcal{A}_{s}$ admits no characters. For example, if we have a (finite unital) semilattice $S$, a family of algebras $\left\{\mathcal{A}_{s}\right\}_{s \in S}$ each having no characters, and a system $\left\{\eta_{t}^{s}: s, t \in S, s \geq t\right\}$ of homomorphisms, we can make $\ell^{1}-\bigoplus_{s \in S} \mathcal{A}_{s}$ into a Banach algebra by setting $a_{s} a_{t}=\eta_{s t}^{s}\left(a_{s}\right) \eta_{s t}^{t}\left(a_{t}\right)$ for $a_{s}$ in $\mathcal{A}_{s}$ and $a_{t}$ in $\mathcal{A}_{t}$. (This construction is analagous to that of the Clifford semigroup algebras which will be presented in Section 2.1, below.)

This brings us to the main result of this article.

Theorem 2.2. Let $\mathcal{A}$ be a Banach algebra graded over a finite semilattice $S$ such that each $\mathcal{A}_{s}$ is amenable. If we have either that (LA1) holds, or (LA2) holds, then $\operatorname{AM}(\mathcal{A}) \geq \operatorname{AM}(S)$.

Proof. $\mathcal{A}$ is amenable by the proposition above.

Let us suppose (LA1) holds. We let for each $p$ in $S, \pi_{p}: \mathcal{A} \rightarrow \mathcal{A}_{p}$ the contractive projection. We define for $a, b \in \mathcal{A}, \pi_{p}(a \otimes b)=\pi_{p}(a) \otimes b$ and $(a \otimes b) \pi_{p}=a \otimes \pi_{p}(b)$. Clearly these actions extend linearly and continuously to define $\pi_{p} D$ and $D \pi_{p}$ for any $D \in \mathcal{A} \otimes^{\gamma} \mathcal{A}$.

We let $\left(D_{\alpha}\right)$ be a bounded approximate diagonal for $\mathcal{A}$ and

$$
D=\sum_{(s, t) \in S \times S} d(s, t) \delta_{s} \otimes \delta_{t}
$$

be the unique diagonal for $\ell^{1}(S)$. We will prove that for $p, q \in S$ and $a \in \mathcal{A}^{p}$, $b \in \mathcal{A}^{q}$ that

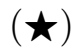

$$
\lim _{\alpha} a m\left(\pi_{p} D_{\alpha} \pi_{q}\right) b=d(p, q) a b .
$$

This requires induction and we will need some preliminary steps.

Suppose that $q \neq p$ in $S$, say $q \nsupseteq p$. If $v_{q} \in \mathcal{A}_{q}$ then (0.2) implies that

$$
\lim _{\alpha} \pi_{p}\left(D_{\alpha} \cdot v_{q}\right) \pi_{q}=\lim _{\alpha} \pi_{p}\left(v_{q} \cdot D_{\alpha}\right) \pi_{q}=0 .
$$

We note that on an elementary tensor in $\mathcal{A} \otimes \mathcal{A}$ we have

$$
m\left(\pi_{p}\left(a \otimes b \cdot v_{q}\right) \pi_{q}\right)=\sum_{t \geq q} \pi_{p}(a) \pi_{t}(b) v_{q}=\sum_{t \geq q} m\left(\pi_{p}(a \otimes b) \pi_{t}\right) v_{q}
$$

Now if $b \in \mathcal{A}^{q}$ we find $v_{q} \in \mathcal{A}_{q}$ and $b^{\prime}$ in $\mathcal{A}^{q}$ such that $b=v_{q} b^{\prime}$ by (2.1). We then have, in analogy to Lemma 1.2 (b), using (2.2) and (2.3)

$$
\lim _{\alpha} \sum_{t \geq q} m\left(\pi_{p} D_{\alpha} \pi_{t}\right) b=\lim _{\alpha} m\left(\pi_{p}\left(D_{\alpha} \cdot v_{q}\right) \pi_{q}\right) b^{\prime}=0 \cdot b^{\prime}=0 .
$$


Similarly we see

$$
\lim _{\alpha} \sum_{s \geq q} b m\left(\pi_{s} D_{\alpha} \pi_{p}\right)=0
$$

Note that if $p, q \in M(S)$ with $p \neq q$, then then $\left(\mathrm{b}_{1}{ }^{\prime}\right)$ takes the form

$$
\lim _{\alpha} m\left(\pi_{p} D_{\alpha} \pi_{q}\right) b=0=d(p, q) b
$$

and a simlar version holds for $\left(\mathrm{b}_{2}{ }^{\prime}\right)$. Thus $(\star)$ holds in this case.

Now we show that for $p \in S$ and $b$ in $\mathcal{A}^{p}$ that

$$
\lim _{\alpha} \pi_{p}\left(m\left(D_{\alpha}\right)\right) b=u(p) b
$$

where $u=\sum_{p \in S} u(p) \delta_{p}$ is the unit for $\ell^{1}(S)$. By (2.1) there are $v_{p}$ in $\mathcal{A}_{p}$ and $b^{\prime}$ in $\mathcal{A}^{p}$ such that $b=v_{p} b^{\prime}$. We have that

$$
\begin{aligned}
v_{p} & =\lim _{\alpha} m\left(D_{\alpha}\right) v_{p}=\lim _{\alpha} \sum_{s \in S} \pi_{s}\left(m\left(D_{\alpha}\right)\right) v_{p} \\
& =\lim _{\alpha}\left[\sum_{s \geq p} \pi_{s}\left(m\left(D_{\alpha}\right)\right) v_{p}+\sum_{s \nsupseteq p} \pi_{s}\left(m\left(D_{\alpha}\right)\right) v_{p}\right]
\end{aligned}
$$

from which it follows that

$$
\lim _{\alpha} \sum_{s \geq p} \pi_{s}\left(m\left(D_{\alpha}\right)\right) v_{p}=\lim _{\alpha} \pi_{p}\left(m\left(D_{\alpha}\right) v_{p}\right)=v_{p}
$$

and hence

$$
\lim _{\alpha} \sum_{s \geq p} \pi_{s}\left(m\left(D_{\alpha}\right)\right) b=\lim _{\alpha} \sum_{s \geq p} \pi_{s}\left(m\left(D_{\alpha}\right)\right) v_{p} b^{\prime}=v_{p} b^{\prime}=b .
$$

In particular, if $p \in M(S)$, then

$$
\lim _{\alpha} \pi_{p}\left(m\left(D_{\alpha}\right)\right) b=b=u(p) b .
$$

Then the equation (2.4) follows inductively from (2.5) and (1.4), using the case of maximal $p$ as a base.

Now we establish an analogue of Lemma1.2(a). For an elementary tensor $a \otimes b$ in $\mathcal{A} \otimes \mathcal{A}$, we have

$$
\pi_{p}(a b)=\sum_{\substack{(s, t) \geq(p, p) \\ s t=p}} \pi_{s}(a) \pi_{t}(b)=\sum_{\substack{(s, t) \geq(p, p) \\ s t=p}} m\left(\pi_{s}(a \otimes b) \pi_{t}\right) .
$$

It then follows from (2.4) and (2.6) that for $b \in \mathcal{A}^{p}$

$$
u(p) b=\lim _{\alpha} \sum_{\substack{(s, t) \geq(p, p) \\ s t=p}} m\left(\pi_{s} D_{\alpha} \pi_{t}\right) b .
$$

Note that if $p \in M(S)$, then by Proposition 1.1, (a') becomes

$$
d(p, p) b=b=\lim _{\alpha} m\left(\pi_{p} D_{\alpha} \pi_{p}\right) b .
$$

Thus $(\star)$ holds in this case. 
We now prove $(\star)$ by induction on pairs $(p, q)$ in $S \times S$ with pairs $(p, q) \in$ $M(S) \times M(S)$ as a base. If $p \in S$, the induction hypothesis is that for $a, b \in \mathcal{A}^{p}$

$$
\lim _{\alpha} a m\left(\pi_{s} D_{\alpha} \pi_{t}\right) b=d(s, t) a b \text { for }(s, t)>(p, p) \text { with } s t=p .
$$

Notice that in the hypothesis above we have $\mathcal{A}^{p} \subset \mathcal{A}^{s} \cap \mathcal{A}^{t}$, and, moreover, either $t \geq s$ or $s \nsupseteq t$. But then it follows from (a') and Lemma 1.2 (a) that

$$
\lim _{\alpha} a m\left(\pi_{p} D_{\alpha} \pi_{p}\right) b=\left[u(p)-\sum_{\substack{(s, t)>(p, p) \\ s t=p}} d(s, t)\right] a b=d(p, p) a b
$$

which establishes $(\star)$ in this case. Also, if $q \neq p$, say $q \nsupseteq p$, then for $a$ in $\mathcal{A}^{p}$ and $b$ in $\mathcal{A}^{q}$ the induction hypothesis is that

$$
\lim _{\alpha} a m\left(\pi_{p} D_{\alpha} \pi_{t}\right) b=d(p, t) a b \text { for } t>q .
$$

Combining this with $\left(\mathrm{b}_{1}{ }^{\prime}\right)$ and Lemma $1.2(\mathrm{~b})$ we obtain the equation $(\star)$ for this case. We can use $\left(b_{2}{ }^{\prime}\right)$ in place of $\left(b_{1}^{\prime}\right)$ above, to acheive $(\star)$ with $p$ and $q$ interchanged.

We now use $(\star)$ to finish the proof. Let for $p, q$ in $S$

$$
\eta(p, q)=\sup _{a \in \mathcal{A}^{p}, b \in \mathcal{A}^{q}} \frac{\|a b\|}{\|a\|\|b\|} .
$$

We note that our assumption (LA1) provides that $\eta(p, q)>0$. For $\varepsilon>0$ let $a_{\varepsilon}$ in $\mathcal{A}^{p}$ and $b_{\varepsilon}$ in $\mathcal{A}^{q}$ be so $\frac{\left\|a_{\varepsilon} b_{\varepsilon}\right\|}{\left\|a_{\varepsilon}\right\|\left\|b_{\varepsilon}\right\|} \geq(1-\varepsilon) \eta(p, q)$. Then by $(\star)$ we have

$$
\begin{aligned}
|d(p, q)|\left\|a_{\varepsilon} b_{\varepsilon}\right\| & =\lim _{\alpha}\left\|a_{\varepsilon} m\left(\pi_{p} D_{\alpha} \pi_{q}\right) b_{\varepsilon}\right\| \leq \liminf _{\alpha}\left\|a_{\varepsilon} m\left(\pi_{p} D_{\alpha} \pi_{q}\right)\right\|\left\|b_{\varepsilon}\right\| \\
& \leq \liminf _{\alpha} \frac{\left\|a_{\varepsilon} m\left(\pi_{p} D_{\alpha} \pi_{q}\right)\right\|}{\left\|a_{\varepsilon}\right\|\left\|m\left(\pi_{p} D_{\alpha} \pi_{q}\right)\right\|}\left\|a_{\varepsilon}\right\|\left\|b_{\varepsilon}\right\|\left\|m\left(\pi_{p} D_{\alpha} \pi_{q}\right)\right\| \\
& \leq \eta(p, q)\left\|a_{\varepsilon}\right\|\left\|b_{\varepsilon}\right\| \liminf _{\alpha}\left\|m\left(\pi_{p} D_{\alpha} \pi_{q}\right)\right\|
\end{aligned}
$$

which implies

$$
(1-\varepsilon)|d(p, q)| \leq \liminf _{\alpha}\left\|m\left(\pi_{p} D_{\alpha} \pi_{q}\right)\right\| \leq \liminf _{\alpha}\left\|\pi_{p} D_{\alpha} \pi_{q}\right\|_{\gamma} .
$$

Thus

$$
\begin{aligned}
\operatorname{AM}(S) & =\sum_{(p, q) \in S \times S}|d(p, q)| \leq \sum_{(p, q) \in S \times S} \liminf _{\alpha}\left\|\pi_{p} D_{\alpha} \pi_{q}\right\|_{\gamma} \\
& \leq \liminf _{\alpha} \sum_{(p, q) \in S \times S}\left\|\pi_{p} D_{\alpha} \pi_{q}\right\|_{\gamma} \stackrel{(\dagger)}{=} \liminf _{\alpha}\left\|D_{\alpha}\right\| \leq \sup _{\alpha}\left\|D_{\alpha}\right\|_{\gamma}
\end{aligned}
$$

where the equality $(\dagger)$ holds because of the isometric identification

$$
\mathcal{A} \otimes^{\gamma} \mathcal{A}=\left(\ell^{1}-\bigoplus_{s \in S} \mathcal{A}_{s}\right) \otimes^{\gamma}\left(\ell^{1}-\bigoplus_{t \in S} \mathcal{A}_{t}\right) \cong \ell^{1}-\bigoplus_{(s, t) \in S \times S} \mathcal{A}_{s} \otimes^{\gamma} \mathcal{A}_{t} .
$$


Thus we have finished the case where we assumed (LA1).

Now suppose we have (LA2). The map

$$
\Pi: \mathcal{A} \rightarrow \ell^{1}(S), \quad \Pi(a)=\sum_{s \in S} \chi_{s}\left(\pi_{s}(a)\right) \delta_{s}
$$

is a contractive homomorphism. Hence it follows that if $\left(D_{\alpha}\right)$ is a bounded approximate diagonal for $\mathcal{A}$ then $\left(\Pi\left(D_{\alpha}\right)\right)$ is an approximate diagonal for $\ell^{1}(S)$. Thus the limit point, i.e. unique cluster point, $D$ of $\left(\Pi\left(D_{\alpha}\right)\right)$ satisfies $\|D\|_{\gamma}=\operatorname{AM}(S)$, whence $\sup _{\alpha}\left\|D_{\alpha}\right\|_{\gamma} \geq \lim _{\alpha}\left\|\Pi\left(D_{\alpha}\right)\right\|_{\gamma} \geq \operatorname{AM}(S)$.

It might seem plausible that in the situation of the theorem above, if it were the case that $\operatorname{AM}\left(\mathcal{A}_{s}\right)=1$, for each $s$, then $\operatorname{AM}(\mathcal{A})=\operatorname{AM}(S)$. Indeed this phenomenon was observed for $S=L_{1}$, in a special case in [14, Theorem 2.3]. However this does not seem to hold in general, as we shall see below.

2.1. Clifford semigroup algebras. Let $S$ be a semilattice, and for each $s$ in $S$ suppose we have a group $G_{s}$, and for each $t \leq s$ a homomorphism $\eta_{t}^{s}: G_{s} \rightarrow G_{t}$ such that for $r \geq s \geq t$ in $S$ we have

$$
\eta_{s}^{s}=\operatorname{id}_{G_{s}} \quad \text { and } \quad \eta_{s}^{r} \circ \eta_{t}^{s}=\eta_{t}^{r}
$$

then $G=\bigsqcup_{s \in S} G_{s}$ (disjoint union) admits a semigroup operation given by

$$
x_{s} y_{t}=\eta_{s t}^{s}\left(x_{s}\right) \eta_{s t}^{t}\left(y_{t}\right)
$$

for $x_{s}$ in $G_{s}$ and $y_{t}$ in $G_{t}$. It is straightforward to check that $G$ is a semigroup, and is called a Clifford semigroup, as such a semigroup was first described in [2]. We note that the set of idempotents $E(G)$ is $\left\{e_{s}\right\}_{s \in S}$, where $e_{s}$ is the neutral element of $G_{s}$, and $E(G)$ is a subsemigroup, isomorphic to $S$. It is clear that

$$
\ell^{1}(G)=\ell^{1}-\bigoplus_{s \in S} \ell^{1}\left(G_{s}\right)
$$

and that $\ell^{1}(G)$ is thus graded over $S$. Note that $\ell^{1}(G)$ satisfies (LA1) by design, and satisfies (LA2) where the augmentation character is used on each $\ell^{1}\left(G_{s}\right)$. As with semilattices we will write $\operatorname{AM}(G)=\operatorname{AM}\left(\ell^{1}(G)\right)$

Consider the semilattice $S=\left\{o, s_{1}, s_{2}, s_{3}, s_{4}, 1\right\}$ whose graph is given below.

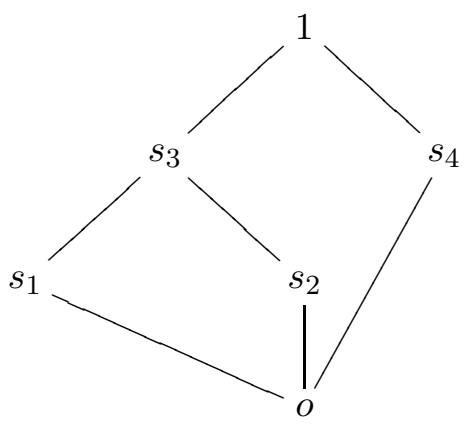


Using the algorithm following Lemma 1.2, with the semilattice ordered as presented, we obtain diagonal $D$ with matrix

$$
[D]=\left[\begin{array}{rrrrrr}
6 & -2 & -2 & 0 & -2 & 1 \\
-2 & 2 & 1 & -1 & 0 & 0 \\
-2 & 1 & 2 & -1 & 0 & 0 \\
0 & -1 & -1 & 2 & 1 & -1 \\
-2 & 0 & 0 & 1 & 2 & -1 \\
1 & 0 & 0 & -1 & -1 & 1
\end{array}\right]
$$

Thus we obtain amenability constant $\operatorname{AM}(S)=41$.

Now let $n \geq 2$ be an integer and $G_{n}$ be the Clifford semigroup graded over $S$ for which

$$
G_{n, s_{3}}=\left\{e_{3}, a, \ldots, a^{n-1}\right\} \quad \text { and } \quad G_{n, s_{i}}=\left\{e_{i}\right\} \text { for all } i \neq 3
$$

and all connecting homomorphisms are trivial. Here, $\left\{e_{3}, a, \ldots, a^{n-1}\right\}$ is a cyclic group, and each other $\left\{e_{i}\right\}$ is the trivial group. This is a finite dimensional commutative amenable algebra, and hence admits a unique diagonal by Proposition 0.1. It is straightforward to verify that if we order the semigroup $\left\{o, e_{1}, e_{2}, e_{3}, a, \ldots, a^{n-1}, e_{4}, 1\right\}$ we obtain matrix for the diagonal

$$
\left[\begin{array}{crrcrcrrr}
6 & -2 & -2 & (1-n) / n & 1 / n & \ldots & 1 / n & -2 & 1 \\
-2 & 2 & 1 & -1 / n & -1 / n & \ldots & -1 / n & 0 & 0 \\
-2 & 1 & 2 & -1 / n & -1 / n & \ldots & -1 / n & 0 & 0 \\
(1-n) / n & -1 / n & -1 / n & (n+1) / n & 0 & \ldots & 0 & 1 & -1 \\
1 / n & -1 / n & -1 / n & 0 & & . \cdot & 1 / n & 0 & 0 \\
\vdots & \vdots & \vdots & \vdots & . \cdot & . \cdot & & \vdots & \vdots \\
1 / n & -1 / n & -1 / n & 0 & 1 / n & & 0 & 0 & 0 \\
-2 & 0 & 0 & 1 & 0 & \ldots & 0 & 2 & -1 \\
1 & 0 & 0 & -1 & 0 & \ldots & 0 & -1 & 1
\end{array}\right] .
$$

Notice that values in positions $\left(o, e_{3}\right), \ldots,\left(o, a^{n-1}\right)$ sum to 0 , the value in the $\left(o, s_{3}\right)$ position in (2.8) above. Similar results holds for all submatirices with indicies from $\left\{e_{3}, a, \ldots, a^{n-1}\right\}$. Summing absolute values of all entries in the matrix we obtain amenability constant $\operatorname{AM}\left(G_{n}\right)=41+4(n-1) / n$. Thus

$$
\operatorname{AM}\left(G_{n}\right)=41+4 \frac{n-1}{n}>41=\operatorname{AM}(S) .
$$

The constant $\operatorname{AM}\left(G_{2}\right)=43$ is the smallest amenability constant we can find for an commutative semigroup which is not of the form $4 n+1$.

2.2. Algebras graded over linear semilattices. We note that if $G$ is a finite Clifford semigroup, graded over a linear semilattice $L_{n}$, then $\operatorname{AM}(G)=$ $\operatorname{AM}\left(L_{n}\right)=4 n+1$. Indeed, this holds more generally, by the following proposition. 
Proposition 2.3. If $\mathcal{A}=\ell_{1}-\bigoplus_{k \in L_{n}} \mathcal{A}_{k}$ is a graded Banach algebra which satisfies (LA1), and $\mathcal{A}_{k}$ is contractible with $\operatorname{AM}\left(\mathcal{A}_{k}\right)=1$ for each $k$ in $L_{n}$, then $\operatorname{AM}(\mathcal{A})=4 n+1$.

Proof. We have from Theorem 2.2 that $\operatorname{AM}(\mathcal{A}) \geq \operatorname{AM}\left(L_{n}\right)=4 n+1$, hence it suffices to exhibit a diagonal $D$ with $\|D\|_{\gamma} \leq 4 n+1$. We will show that such $D$ exists by induction.

Write $L_{n}=\{0,1, \ldots, n\}$. We identify $L_{k}$ as an ideal of $L_{n}$ for each $k=0,1, \ldots, n-1$ in the usual way. Let us note that if $\left(u_{k, \alpha}\right)$ is a bounded approximate identity for $\mathcal{A}_{k}$, which satisfies (LA1), then the unit $e_{k}$ of $\mathcal{A}_{k}$ is the limit point of $\left(u_{k, \alpha}\right)$, and hence $e_{k}$ is the unit for $\mathcal{A}^{k}=\ell^{1}-\bigoplus_{j \in L_{k}} \mathcal{A}_{j}$. Note, moreover, that the assumption that $\operatorname{AM}\left(\mathcal{A}_{k}\right)=1$ forces $\left\|e_{k}\right\|=1$.

Let $\varepsilon>0$. Suppose for $k<n$ we have a diagonal $D^{k}$ for $\mathcal{A}^{k}$ with $\left\|D^{k}\right\|_{\gamma}<4 k+1+\varepsilon$. For $k=0$, such a diagonal exists as $\operatorname{AM}\left(\mathcal{A}_{0}\right)=1$. We let

$$
D_{k+1}=\sum_{i=1}^{\infty} a_{i} \otimes b_{i}, \quad a_{i}, b_{i} \in \mathcal{A}_{k+1}
$$

be a diagonal for $\mathcal{A}_{k+1}$ with $\left\|D_{k+1}\right\|_{\gamma} \leq \sum_{i=1}^{\infty}\left\|a_{i}\right\|\left\|b_{i}\right\|<1+\varepsilon$. We then set

$$
D^{k+1}=\sum_{i=1}^{\infty} a_{i} \cdot\left(\left(e_{k+1}-e_{k}\right) \otimes\left(e_{k+1}-e_{k}\right)+D^{k}\right) \cdot b_{i} .
$$

Clearly

$$
\left\|D^{k+1}\right\|_{\gamma} \leq(4+(4 k+1+\varepsilon))(1+\varepsilon)=4(k+1)+1+O(\varepsilon) .
$$

Applying the multiplication map, and noting that $m\left(D^{k}\right)=e_{k}$, we have

$$
\begin{aligned}
m\left(D^{k+1}\right) & =\sum_{i=1}^{\infty} a_{i}\left(e_{k+1}-e_{k}-e_{k}+e_{k}+m\left(D^{k}\right)\right) b_{i} \\
& =\sum_{i=1}^{\infty} a_{i} e_{k+1} b_{i}=m\left(D_{k+1}\right)=e_{k+1}
\end{aligned}
$$

so (0.3) for $D^{k+1}$ is satisfied. Now if $a \in \mathcal{A}_{k+1}$ then by property (0.4) for $D_{k+1}$ we have $\sum_{i=1}^{\infty}\left(a a_{i}\right) \otimes b_{i}=\sum_{i=1}^{\infty} a_{i} \otimes\left(b_{i} a\right)$, so it follows that $a \cdot D^{k+1}=$ $D^{k+1} \cdot a$. Now if $a \in \mathcal{A}^{k}$, then each $a a_{i} \in \mathcal{A}^{k}$ so

$$
\begin{aligned}
a \cdot D^{k+1} & =\sum_{i=1}^{\infty}\left(a a_{i}\right) \cdot\left(\left(e_{k+1}-e_{k}\right) \otimes\left(e_{k+1}-e_{k}\right)+D^{k}\right) \cdot b_{i} \\
& =\sum_{i=1}^{\infty}\left(\left[a a_{i}\left(e_{k+1}-e_{k}\right)\right] \otimes\left(e_{k+1}-e_{k}\right)+\left(a a_{i}\right) \cdot D^{k}\right) \cdot b_{i} \\
& =\sum_{i=1}^{\infty} D^{k} \cdot\left(a a_{i} b_{i}\right)=D^{k} \cdot a=a \cdot D^{k}
\end{aligned}
$$


which, by symmetric argument, is exactly the value of $D^{k+1} \cdot a$. Since any $a \in \mathcal{A}^{k+1}$ is a sum $a=\pi_{k+1}(a)+\left(a-\pi_{k+1}(a)\right)$ where, $\pi_{k+1}(a) \in \mathcal{A}_{k+1}$ and $a-\pi_{k+1}(a) \in \mathcal{A}^{k}$, we obtain (0.4) for $D^{k+1}$.

We note that to generalise our proof of the preceding result to amenable but not contractible Banach algebras, we would require at each stage approximate diagonals $D_{\alpha}^{k}$ such that $\left\|m\left(D_{\alpha}^{k}\right)\right\|=1$, which we do not know how to construct, in general. We point the reader to [13, Theorem 2.3] to see a computation performed on a Banach algebra graded over $L_{1}$.

We note that we can modify the proof of Proposition 2.3 to see that $a$ Banach algebra $\mathcal{A}=\ell_{1}-\bigoplus_{s \in F_{2}^{1}} \mathcal{A}_{s}$ graded over $F_{2}^{1}$, where each $\mathcal{A}_{s}$ is contractible with $\operatorname{AM}\left(\mathcal{A}_{s}\right)=1$, satisfies $\operatorname{AM}(\mathcal{A}) \leq 45$. This is larger than $\operatorname{AM}\left(F_{2}^{1}\right)=25$ from Example 1.4. We have found no examples of such Banach algebras $\mathcal{A}$ with $\operatorname{AM}(\mathcal{A})>25$. However, we conjecture only for semilattices $S=L_{n}$, that a Banach algebra $\mathcal{A}=\ell_{1}-\bigoplus_{s \in S} \mathcal{A}_{s}$ graded over $S$, where each $\mathcal{A}_{s}$ is amenable with $\operatorname{AM}\left(\mathcal{A}_{s}\right)=1$, satisfies $\operatorname{AM}(\mathcal{A})=\operatorname{AM}(S)$. It would be interesting to find non-linear unital semilattices over which this conjecture holds.

2.3. On allowable amenability constants. We close by partially answering a question posed in [3]. There it is proved, that there is no semigroup $G$ such that $1<\operatorname{AM}(G)<5$. It is further conjectured that there are no semigroups $G$ for which $\operatorname{AM}(G) \in(5,7) \cup(7,9)$. In 3 , there is an example given of a noncommutative semigroup $G$ with $\operatorname{AM}(G)=7$. For commutative semigroups there is a further gap.

Proposition 2.4. There is no commutative semigroup $G$ such that

$$
5<\operatorname{AM}(G)<9 .
$$

Proof. Since $G$ is commutative, it is proved in [5, Theorem 2.7] that if $\ell^{1}(G)$ is amenable, then $G$ is a Clifford semigroup, whose component groups are abelian, graded over a finite semilattice $S$. If $\operatorname{AM}(G)<9$, then by Theorem 2.2 then $\operatorname{AM}(S)<9$ and hence by Theorem 1.7 and the corollary which follows it we have

$$
2|S|-1 \leq \operatorname{AM}(S) \leq 5
$$

so $|S| \leq 3$. Clearly, if $|S|=1, S=L_{0}$, and if $|S|=2, S=L_{1}$. If $|S|=3$ then $S$ is either unital, in which case $S=L_{2}$, or $S$ has 2 maximal elements, in which case $S=F_{2}$; in either case $\operatorname{AM}(S)=9$, contradicting our assumptions. Thus $S=L_{0}$ or $L_{1}$. But it then follows by a straighforward adaptation of [13, Theorem 2.3] that $\operatorname{AM}(G)=1$ or 5 . In particular $\operatorname{AM}(G) \leq 5$.

Acknowledgements. The authors are grateful to H.G. Dales for valuable questions and discussion, and the Y. Choi for providing a preprint of his article [1]. 


\section{REFERENCES}

[1] Y. Choi. Biflatness of $\ell^{1}$-semilattice algebras. To appear in Semigroup Forum, see ArXiv math.FA/0606366.

[2] A. H. Clifford. Semigroups admitting relative inverses. Ann. Math., 42:1037-1049, 1941.

[3] H. G. Dales, A. T.-M. Lau, and D. Strauss. Banach algebras on semigroups and their compactifications. Manuscript, 2006.

[4] J. Duncan and I. Namioka. Amenability of inverse semigroups and their semigroup algebras. Proc. Roy. Soc. Edinburgh Sect. A, 80:309-321, 1978.

[5] N. Grønbæk. Amenability of discrete convolution algebras, the commutative case. Pacific J. Math., 143:243-249, 1990.

[6] E. Hewitt and K. A. Ross. Abstract Harmonic Analysis II, volume 152 of Grundlehern der mathemarischen Wissenschaften. Springer, New York, 1970.

[7] M. Ilie and N. Spronk. The spine of a Fourier-Stieltjes algebra. Proc. London Math. Soc. (3), 94:273-301, 2004.

[8] M. Ilie and N. Spronk. The algebra generated by idempotents in a Fourier-Stieltjes algebra. To appear in Houston Math. J., see Arxiv math.FA/0510514, 2005.

[9] J. Inoue. Some closed subalgebras of measure algebras and a generalization of P.J. Cohen's theorem. J. Math. Soc. Japan, 23:278-294, 1971.

[10] B. E. Johnson. Approximate diagonals and cohomology of certain annihilator Banach algebras. AMer. J. Math, 94:685-698, 1972.

[11] B. E. Johnson. Non-amenability of the Fourier algebra of a compact group. J. London Math. Soc., 50:361-374, 1994.

[12] V. Runde. Lectures on Amenability, volume 1774 of Lec. Notes in Math. Springer, Berlin Heildelberg, 2002.

[13] V. Runde and N. Spronk. Operator amenability of Fourier-Stieltjes algebras. Math. Proc. Camb. Phil. Soc., 136:675-686, 2004.

[14] V. Runde and N. Spronk. Operator amenability of Fourier-Stieltjes algebras II. To appear in Bull. London Math. Soc., see ArXiv math.FA/0507373, 2005.

[15] R.P. Stanley. Enumerative Combinatorics, volume I. Wadsworth \& Brooks, Belmont, California, 1986.

[16] R. Stokke. Approximate diagonals and følner conditions for amenable group and semigroup algebras. Studia. Math., 164:139-159, 2004.

[17] J. L. Taylor. Measure Algebras, volume 16 of Conference Board of Mathematical Sciences. American Mathematical Society, Providence, RI, 1973.

Mahya Ghandehari

Address: Department of Pure Mathematics, University of Waterloo, Waterloo, ON N2L 3G1, Canada

E-mail: mghandeh@uwaterloo.ca

Hamed Hatami

Address: Department of Computer Science, University of Toronto, Toronto, ON M5S 3G4, Canada

E-mail:hamed@cs.toronto.ca

Nico Spronk

Address: Department of Pure Mathematics, University of WAterloo, Waterloo, ON N2L 3G1, Canada

E-mail: nspronk@uwaterloo.ca 